\title{
Características psicométricas de la adaptación colombiana del Cuestionario de Agresividad de Buss y Perry en una muestra de preadolescentes y adolescentes de Bucaramanga*
}

\author{
Psychometric Properties of Colombian Adaptation of Buss and Perry's
} Aggression Questionnaire for Teenagers in a Sample of Bucaramanga

Recibido: febrero 17 de 2011 | Revisado: julio 21 de 2011 | Aceptado: septiembre 9 de 2011

\author{
Nicolás CHAHÍN-PinZÓN ** \\ URBANO LORENZO-SEVA *** \\ ANDREU VIGIL-COLET ***** \\ Universidad Rovira i Virgili, Tarragona, España
}

SICI: 1657-9267(201209)11:3<979:CCABPB>2.0.TX;2-9

Para citar este artículo: Chahín-Pinzón, N., Lorenzo-Seva, U. \& Vigil-Colet, A. (2012). Características psicométricas de la adaptación colombiana del Cuestionario de Agresividad de Buss y Perry en una muestra de preadolescentes y adolescentes de Bucaramanga. Universitas Psychologica, 11(3), 979. 988.

* Agradecimientos: Esta investigación ha estado financiada por una beca del Ministerio Español de Educación y Ciencia (PSI2008-00236/PSIC).

*** Doctorando programa de doctorado en Evaluación y Medida de la Conducta de la Universidad Rovira i Virgili. E-mail: nicocha@yahoo.com

**** Catedrático del Área de Metodología de las Ciencias del Comportamiento. Departamento de Psicología. E-mail: urbano.lorenzo@urv.cat. ResearcherID: Lorenzo-Seva, U. G-4228-2011.

***** Catedrático del Área de Metodología de las Ciencias del Comportamiento. Departamento de Psicología. E-mail: andreu.vigil@urv.cat. ResearcherID:VigilColet, A. B-8650-2011.
RESUMEN

Este estudio presenta la adaptación colombiana del Cuestionario de Agresividad de Buss y Perry para preadolescentes y adolescentes, desarrollado a partir de la versión española abreviada de 20 ítems. Dicha adaptación se administró a una muestra de 535 niños (269 niños y 266 niñas) con un rango de edades de 8-16 años, pertenecientes a tres colegios del área metropolitana de Bucaramanga. Los resultados del análisis factorial confirmatorio indican un buen ajuste al modelo de cuatro factores que ha sido descrita para otros idiomas y adaptaciones. El test presentó una fiabilidad satisfactoria para la escala total $(a=0.82)$ y para la escala de agresividad física $(a=0.75)$, mientras que para las demás escalas varía en función de la edad, no siendo recomendable su aplicación en edades inferiores a los 12 años. Al analizar los efectos del sexo sobre la agresividad, se verificó que dicho efecto se debía exclusivamente a las diferencias en agresividad física. Finalmente, se pone de manifiesto la importancia de considerar los aspectos culturales y lingüísticos en la adaptación de un instrumento, incluso, como en este caso, cuando se trata del mismo idioma.

Palabras clave autores:

Agresividad, adaptación de escalas, adolescencia.

Palabras clave descriptores:

Adaptación de test, pruebas psicológicas, investigación cuantitativa.

\footnotetext{
A B S T R A C T

This study presents the Colombian adaptation of the Buss and Perry's Aggression Questionnaire for preadolescents and adolescents. The test was administered to a sample of 535 children (269 boys and 266 girls) with an age range of 8-16 years old, belonging to three schools of Bucaramanga. Confirmatory factor analysis showed a good fit to the four-factor model and reliability was satisfactory. The reliability was good for overall scale $(\alpha=0.82)$ and physical aggression scale $(a=0.75)$ while it was sufficient or poor for the other scales depending upon the age group, not being suitable for ages under 12 years-old. Referring to sex effects, we found them only for physical aggression. Finally, this study places particular emphasis on the importance of linguistic and cultural aspects in test adaptation, even when both cultures share the same language. Key words authors: Aggression, Scale Adaptation, Adolescence.

Key words plus:

Test Adaptation, Psychological Tests, Quantitative Research.
} 


\section{Introducción}

El Cuestionario de Agresividad (AQ) de Buss y Perry (1992) es, en la actualidad, uno de los instrumentos psicométricos más utilizados para evaluar e investigar la conducta agresiva. El AQ se desarrolló a partir del Hostility Inventory de Buss y Durkee (1957), que comprendía siete escalas y 75 ítems. Pero sus limitaciones psicométricas llevaron a Buss y Perry a una versión más refinada, compuesta 29 ítems, con una estructura factorial que comprende cuatro escalas de agresividad: física y verbal, que representan los componentes instrumental y motor; ira, el componente afectivo; y hostilidad, el componente cognitivo de la agresión. Su estructura factorial ha sido estudiada también en diversos idiomas y culturas, como es el caso, entre otras, de la versión japonesa (Nakano, 2001; Ramírez, Andreu \& Fujihara, 2001), eslovaca (Lovas \& Trenkova, 1996), alemana (Von Collani \& Werner, 2005), holandesa (Meesters, Muris, Bosma, Schouten \& Beuving, 1996), francesa (Pfister \& Masse, 2001), italiana (Fossati, Maffei, Acquarini \& Di Ceglie, 2003) y española (Andreu, Peña \& Graña, 2002; García-León et al., 2002).

A pesar de la elevada coincidencia de numerosos estudios en la solución de los cuatro factores, diversos investigadores han encontrado problemas en el ajuste de algunos ítems, especialmente los revertidos y los pertenecientes a la Escala de Hostilidad (Bernstein \& Gesn, 1997; Harris, 1995; Meesters et al., 1996). Teniendo en cuenta que estos ajustes afectaban a distintos ítems en las diversas adaptaciones del cuestionario, se desarrolló una versión del AQ para el contexto español que eliminaba aquellos ítems que presentaban una baja congruencia a través de las distintas culturas (Vigil-Colet, Lorenzo-Seva, Codorniu-Raga \& Morales, 2005). Como resultado, se obtuvo una nueva versión reducida para adultos de 20 ítems que presenta un ajuste satisfactorio cuando ha sido evaluada mediante análisis factorial confirmatorio. Además, por el procedimiento que se ha llevado a cabo se garantiza una mayor validez transcultural que permite llevar a cabo comparaciones de los resultados entre diferentes culturas (Morales-Vives, Codorniu-Raga \& Vigil-Colet, 2005).
Un aspecto muy importante para tener presente acerca del AQ, es que a pesar de que fue desarrollado inicialmente para ser administrado en adultos, ha señalado su adecuación en poblaciones jóvenes. Es el caso de la adaptación para adolescentes y preadolescentes desarrollada por Santisteban, Alvarado y Recio (2007) a partir de la versión original del AQ, que ha mostrado una adecuada consistencia interna y un buen ajuste al modelo de cuatro factores. Lo que indica que este puede ser útil para obtener estimaciones fiables y válidas de la agresividad en estos rangos de edad (Santisteban \& Alvarado, 2009). Por otra parte, también se ha encontrado en Asia que la versión abreviada del AQ de 12 ítems, presenta características similares en adolescentes (Ang, 2007).

En América Latina, el AQ ha tenido solo dos adaptaciones, ambas para adultos. En la primera, desarrollada en Colombia por Castrillón, Ortiz y Vieco (2004), se encontraron cinco factores, añadiendo el factor de No Agresión a los cuatro descritos originalmente; además en el factor de hostilidad se encontraron diferencias respecto a estudios anteriores. Los propios autores puntualizaron que las diferencias encontradas podían deberse a efectos culturales. Por otra parte, hay que señalar que este estudio no partió la versión original del $A Q$, ya que a esta se le agregaron 11 ítems. En la segunda adaptación, realizada en El Salvador, se encontró la misma estructura factorial del AQ original, aunque los factores presentaron ligeras diferencias en su composición y dos de los ítems debieron ser eliminados por su baja comunalidad (Sierra \& Gutiérrez, 2007).

Debido a que en Latinoamérica no existe en la actualidad una versión del AQ para preadolescentes y adolescentes, se decidió realizar en esta investigación una adaptación para dicha población, a partir de la versión reducida para adultos desarrollada en España (Morales-Vives et al., 2005). Se consideró que esta tiene la ventaja adicional de haber sido desarrollada teniendo en cuenta características transculturales, que se encuentra libre de sesgos debidos al sexo y que su aplicación es mucho más rápida ya que posee un número menor de ítems (Condon, Morales- 
Vives, Ferrando \& Vigil-Colet, 2006; Vigil-Colet et al., 2005).

Sin embargo, realizar una adaptación del AQ desde dos contextos tan distintos, como lo son España y Colombia, requiere no perder de vista algunos aspectos previos. Aunque la adaptación de pruebas por lo general implica traducción de un idioma a otro, esta va mucho más allá de una simple traducción, ya que implica el análisis de las diferencias culturales y de las variedades lingüísticas de la cultura original y de aquella a la que se pretende adaptar el instrumento (Hambleton, 2005). Este análisis de las diferencias culturales y lingüísticas es un aspecto clave en varias de las directrices propuestas por la Comisión Internacional de Tests (ITC) para la adaptación de tests en distintas culturas (Hambleton, 1994; van de Vijver \& Hambleton, 1996). No obstante, es muy frecuente el caso en el que un instrumento adaptado o desarrollado en España, por el hecho de estar en idioma español se asuma a priori que sus propiedades psicométricas van a ser las mismas al ser aplicado en cualquier otro país de habla hispana. Esta idea no considera que las variantes lingüísticas y las diferencias culturales afectan el funcionamiento del instrumento de medida. Es así como una misma expresión puede implicar un nivel distinto del rasgo que se pretende medir en función de cada país. Por ejemplo, "amenazar a alguien" no implica en España una alta agresividad, mientras que en Colombia significa un nivel muy elevado de agresividad, en el que está en riesgo la vida del individuo amenazado. Por lo argumentado, se considera que la aplicación del AQ en población preadolescente y adolescente, requiere previamente de un proceso serio de adaptación específico, en lugar de aplicar directamente la versión española.

Por tanto, el objetivo de este estudio es el desarrollo de una versión reducida del AQ ajustada a las características de la población preadolescente y adolescente colombiana, a partir de la realizada en el contexto español (Morales-Vives et al., 2005). En esta nueva adaptación, se estudiarán sus propiedades psicométricas y su estructura factorial. Por otra parte, y considerando la especial importancia de la evaluación de la agresividad en estas edades, se pretende igualmente analizar sus propiedades en los diferentes grupos de edad.

\section{Método}

\section{Participantes}

A partir de una muestra inicial de 616 sujetos se eliminaron aquellos casos en los que faltaba la respuesta a algún ítem, obteniéndose una muestra final constituida por 535 sujetos. Las edades de la muestra oscilan entre 8 y 16 años $(M=12.74$, $D E=2.111)$, donde 266 (49.72\%) son mujeres $(\mathrm{M}=12.94, \mathrm{DE}=2.095)$ y $269(50.28 \%)$ hombres $(\mathrm{M}=12.54, \mathrm{DE}=2.112)$. La diferencia entre la edad promedio de hombres y mujeres resultó significativa $(t=2.224, p=0.027)$, aunque el tamaño del efecto extremadamente bajo $(d=0.09)$ permite considerar a ambos grupos prácticamente equivalentes en edad. La muestra está compuesta por estudiantes de los grados $4^{\circ}$ al $11^{\circ}$. Los participantes pertenecen a tres colegios situados en el área metropolitana de Bucaramanga (Colombia), Centro Piloto Simón Bolívar $(N=240)$, Instituto INEM Custodio García Rovira $(N=114)$ y Colegio Gimnasio Superior $(N=181)$, los dos primeros de carácter oficial y el último, privado. La distribución de la muestra por rango de edades, sexo y grado puede apreciarse en la Tabla 1.

\section{Procedimiento}

Inicialmente se procedió a contactar con los rectores, coordinadores de jornada, directores de curso y psico-orientadores de los tres centros educativos seleccionados, con el fin de concertar las entrevistas para explicar los objetivos y procedimientos que se llevarían a cabo, y obtener su autorización y la de los padres. Una vez obtenidos los permisos respectivos, en presencia de cada profesor se informó a los estudiantes sobre la actividad que se desarrollaría. La participación fue totalmente voluntaria. El cuestionario fue aplicado por un psicólogo acompañado por un profesor, dentro del horario de clase, en grupos de 30 personas, en los salones donde habitualmente se tienen las actividades escolares. Las instrucciones, condiciones de 
TABLA 1

Distribución de la muestra por edades, sexo y grado

\begin{tabular}{|c|c|c|c|c|c|c|c|c|c|c|c|}
\hline & \multirow[t]{2}{*}{ Edad } & \multirow[b]{2}{*}{ Col } & \multicolumn{8}{|c|}{ Grado* } & \multirow[t]{2}{*}{ Total } \\
\hline & & & 4 & 5 & 6 & 7 & 8 & 9 & 10 & 11 & \\
\hline & & Es & \multicolumn{2}{|c|}{ Primaria } & \multicolumn{4}{|c|}{ Secundaria-Obligatoria } & \multicolumn{2}{|c|}{ Bachillerato } & \\
\hline \multirow{4}{*}{ 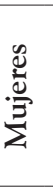 } & $8-10$ & & 13 & 15 & 4 & 0 & 0 & 0 & 0 & 0 & 32 \\
\hline & $11-13$ & & 4 & 7 & 57 & 11 & 21 & 21 & 0 & 0 & 121 \\
\hline & $14-16$ & & 0 & 0 & 1 & 1 & 6 & 40 & 28 & 37 & 113 \\
\hline & Total & & 17 & 22 & 62 & 12 & 27 & 61 & 28 & 37 & 266 \\
\hline \multirow{4}{*}{ 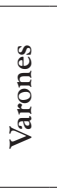 } & $8-10$ & & 20 & 27 & 7 & 0 & 0 & 0 & 0 & 0 & 54 \\
\hline & $11-13$ & & 6 & 10 & 56 & 18 & 13 & 13 & 0 & 0 & 116 \\
\hline & $14-16$ & & 0 & 0 & 0 & 1 & 17 & 32 & 31 & 18 & 99 \\
\hline & Total & & 26 & 37 & 63 & 19 & 30 & 45 & 31 & 18 & 269 \\
\hline \multirow{4}{*}{ 泀 } & $8-10$ & & 33 & 42 & 11 & 0 & 0 & 0 & 0 & 0 & 86 \\
\hline & $11-13$ & & 10 & 17 & 113 & 29 & 34 & 34 & 0 & 0 & 237 \\
\hline & $14-16$ & & 0 & 0 & 1 & 2 & 23 & 72 & 59 & 55 & 212 \\
\hline & Total & & 43 & 59 & 125 & 31 & 57 & 106 & 59 & 55 & 535 \\
\hline
\end{tabular}

*Se dan los grados para Colombia (Col) y su equivalencia para el sistema educativo de España (Es).

Fuente: elaboración propia.

aplicación y manejo de la información, garantizaban la confidencialidad y el anonimato de todos los datos. El tiempo para resolver el cuestionario osciló entre los 12 y 15 minutos.

\section{Instrumentos}

Versión reducida del cuestionario AQ de Buss y Perry en español (Vigil-Colet et al., 2005), compuesto por 20 ítems en escala tipo Likert con cinco alternativas $(1=$ Muy rara vez; $5=$ Muy frecuentemente). Posee cuatro escalas: agresividad física (7 ítems), agresividad verbal (4 ítems), ira (4 ítems) y hostilidad (5 ítems). La fiabilidad de las mismas es de $a=0.88 ; 0.71 ; 0.68$ y 0.65 , respectivamente, mientras que para la escala total es de $a=0.87$ (Morales-Vives et al., 2005).

Con el fin de adaptar dicho instrumento para su administración en Colombia, un psicólogo colombiano y dos españoles con experiencia en la adaptación de cuestionarios analizaron su adecuación cultural y lingüística. Dicho análisis implicó modificar en mayor o menor medida 16 de los 20 ítems, bien por diferencias culturales o lingüísticas, o para adaptar su contenido a este tipo de población. Así por ejemplo, se modificaron expresiones como: "me incitan" por "me provocan", "recurrir a la violencia" por "pelear" o "me enfado rápidamente" por "me da rabia fácilmente", entre otras.

El cuestionario resultante en una segunda fase fue evaluado por ocho licenciados colombianos dedicados a la enseñanza, en relación a la facilidad de comprensión de los ítems por parte de sus alumnos. Los conceptos de los docentes llevaron a unos cuantos cambios ligeros en algunos de los ítems, por ejemplo se sustituyó "golpear" por "pegar" y "provocar" por "molestar". Finalizada esta fase, se obtuvo la versión definitiva del AQ para ser administrada en la muestra seleccionada de preadolescentes y adolescentes. La Tabla 2 muestra esta versión junto a la original española.

\section{Análisis de datos}

Partiendo de que el AQ posee una estructura factorial que ha sido ampliamente replicada en numerosos idiomas y culturas, se ha considerado que la aproximación más adecuada para analizar la estructura factorial de esta nueva versión sea de tipo confirmatorio. Para tal fin, se ha adoptando la estructura de cuatro factores de las versiones española y anglosajona como hipótesis inicial. 
TABLA 2

Ítems del AQ de la versión española y de la adaptación colombiana para preadolescentes y adolescentes

\begin{tabular}{|c|c|c|}
\hline Escala & Versión española & Versión \\
\hline \multirow{7}{*}{ 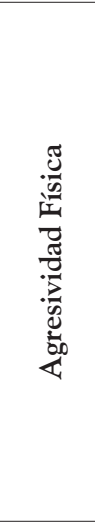 } & $\begin{array}{l}\text { De vez en cuando no puedo controlar el impulso de } \\
\text { golpear a otra persona. }\end{array}$ & $\begin{array}{l}\text { De vez en cuando no puedo controlar el impulso de } \\
\text { golpear a otra persona. }\end{array}$ \\
\hline & $\begin{array}{l}\text { Si se me provoca lo suficiente, puedo golpear a otra } \\
\text { persona. }\end{array}$ & $\begin{array}{l}\text { Si me molestan mucho, puedo llegar a pegarle a otra } \\
\text { persona. }\end{array}$ \\
\hline & $\begin{array}{l}\text { Si alguien me golpea, le respondo golpeándole } \\
\text { también. }\end{array}$ & Si me pegan, yo devuelvo el golpe. \\
\hline & $\begin{array}{l}\text { Si tengo que recurrir a la violencia para proteger mis } \\
\text { derechos, lo hago. }\end{array}$ & $\begin{array}{l}\text { Sit } \\
\text { hag }\end{array}$ \\
\hline & ue me incita hasta tal punto que llegamos & estan tanto que \\
\hline & ado a gente que conozco. & He intimidado a personas que conozco. \\
\hline & a estar tan furioso que rompía cosas. & Cuando me han "sacado la piedra" he dañado cosas. \\
\hline \multirow{4}{*}{ 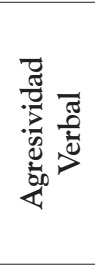 } & uerdo con mis amigos, discuto & ho estoy de ac \\
\hline & A menudo no estoy de acuerdo con la gente. & uencia no estoy de acuerdo con la gente. \\
\hline & $\begin{array}{l}\text { Cuan } \\
\text { pued }\end{array}$ & $\begin{array}{l}\text { Cuar } \\
\text { pued }\end{array}$ \\
\hline & Mis a & Mis \\
\hline \multirow{4}{*}{$\cong$} & o rápidamente, pero se me pasa enseguida. & Me da rabia fácilmente, pero se me pasa rápido. \\
\hline & Cuando estoy frustrado, muestro el enfado que tengo. & Cuando tengo rabia, no la disimulo. \\
\hline & fadado como si & $\begin{array}{l}\text { ia que me siento } c \\
\text { r. }\end{array}$ \\
\hline & Algunas veces pierdo los $\mathrm{e}$ & Algunas veces se me "salta la piedra" sin razón. \\
\hline \multirow{5}{*}{ 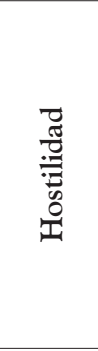 } & A veces soy bastante envidioso. & A veces soy bastante envidioso. \\
\hline & $\begin{array}{l}\text { r qué algunas veces me siento tan } \\
\text { zunas cosas. }\end{array}$ & $\begin{array}{l}\text { o por qué me siento tan } \\
\text { s. }\end{array}$ \\
\hline & Sé que mis amigos me critican a mis espaldas. & Sé que mis amigos me critican a mis espaldas. \\
\hline & $\begin{array}{l}\text { Cuando la gente se muestra especialmente amigable, } \\
\text { me pregunto qué querrán. }\end{array}$ & $\begin{array}{l}\text { Cuando las personas se muestran muy amigables, me } \\
\text { pregunto qué es lo que quieren. }\end{array}$ \\
\hline & $\begin{array}{l}\text { Algunas ocasiones siento que la gente se está riendo } \\
\text { de mí a mis espaldas. }\end{array}$ & $\begin{array}{l}\text { A veces siento que la gente se ríe de mí a mis } \\
\text { espaldas. }\end{array}$ \\
\hline
\end{tabular}

Fuente: elaboración propia.

Los índices de ajuste utilizados para evaluar el análisis factorial confirmatorio (AFC) serán los propuestos por Hu y Bentler (1999), como lo son el índice de ajuste comparativo (CFI), el índice de ajuste no normativo (NFI) y la raíz cuadrada media de error de aproximación (RMSEA). Aunque no existe un consenso absoluto, se consideran habitualmente aceptables valores de corte iguales o superiores a 0.9 para los dos primeros, mientras que para el RMSEA son aceptables valores inferiores a 0.08 y excelentes los iguales o inferiores a 0.05 (Bentler, 1990; Fan \& Sivo, 2007). El AFC se llevó a cabo utilizando el software Mplus 5.1. (Muthen \& Muthen, 2008) sobre la matriz de correlaciones policóricas, debido a que las respuestas a los ítems pueden considerarse como pertenecientes a una escala de medida ordinal.

En caso que la estructura factorial se confirmara, se analizaría la fiabilidad de las escalas, así como la puntuación total mediante el índice alfa de Cronbach. Este análisis se llevaría a cabo para toda la muestra y para los distintos grupos de edad, con el fin de determinar a partir de qué edad puede considerarse apropiada la utilización del AQ 
en esta versión. Estos análisis junto con el cálculo de los estadísticos descriptivos y el análisis de los efectos de edad y sexo, se llevaron a cabo utilizando el software SPSS 17.0.

\section{Resultados}

El AFC planteado para la estructura de cuatro factores proporcionó los siguientes índices de ajuste del modelo: $\mathrm{CFI}=0.92, \mathrm{NFI}=0.90 \mathrm{y}$ RMSEA $=0.049$ (I.C. al $90 \%: 0.044$ - 0.057), con una probabilidad $p=0.349$ de que el valor poblacional de RMSEA sea inferior a 0.05. De este modo, y teniendo en cuenta los puntos de corte de estos indicadores expuestos con anterioridad, puede considerarse que todos ellos muestran un ajuste más que aceptable del modelo, siendo excelente en el caso del RMSEA. Por consiguiente, este análisis indica la adecuación de la adaptación colombiana del AQ para preadolescentes y adolescentes a la estructura de cuatro factores, replicándose los factores de Agresividad Física, Agresividad Verbal, Ira y Hostilidad descritos tanto para la versión original como para las subsiguientes adaptaciones a otros idiomas y versiones.

Una vez establecida la estructura factorial, se calcularon los estadísticos descriptivos de las es- calas. La Tabla 3 muestra los estadísticos de las cuatro escalas para los grupos de sexo y para los tres grupos de edad.

El análisis multivariado de la varianza utilizando los grupos de edad y el sexo como factores, mostró la inexistencia de efectos tanto de la variable edad como de la interacción Edad x Sexo sobre las escalas del AQ. En cambio, el análisis sí mostró efectos significativos de la variable sexo sobre las escalas del AQ $\left(F_{(4,485)}=9.72 ; p<0.01\right) . Y$ al analizar los efectos del sexo sobre cada una de las cuatro escalas a nivel univariado, se verificó que dicho efecto se debía exclusivamente a las diferencias de sexo en agresividad física $\left(F_{(1,488)}=29.15\right.$; $p<0.01)$, presentando los jóvenes puntuaciones superiores $(d=0.64)$.

La Tabla 4 muestra las fiabilidades obtenidas para el total de la muestra y para los distintos grupos de edad. Tal y como se puede comprobar, solo la escala de Agresividad Física y la escala total presentan coeficientes de fiabilidad adecuados en todos los grupos de edad. Por su parte, en el grupo de edad mayor (14 - 16 años) las escalas de Agresividad Verbal, Ira y Hostilidad presentan coeficientes que podrían considerarse como marginalmente aceptables, puesto que el valor $\mathrm{a}=0.70$, generalmente considerado como aceptable, se encuentra

TABLA 3

Estadísticos descriptivos del AQ de la adaptación colombiana desglosados por sexo y edad

\begin{tabular}{|c|c|c|c|c|c|c|c|c|c|}
\hline & & \multicolumn{4}{|c|}{ Varones } & \multicolumn{4}{|c|}{ Mujeres } \\
\hline & & \multicolumn{2}{|c|}{ Media } & \multicolumn{2}{|c|}{$D E$} & \multicolumn{2}{|c|}{ Media } & \multicolumn{2}{|c|}{$\mathrm{DE}$} \\
\hline \multirow{8}{*}{ 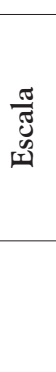 } & Física & \multicolumn{2}{|c|}{17.98} & \multicolumn{2}{|c|}{5.6} & \multicolumn{2}{|c|}{14.57} & \multicolumn{2}{|c|}{5.1} \\
\hline & Verbal & \multicolumn{2}{|c|}{9.19} & \multicolumn{2}{|c|}{3} & \multicolumn{2}{|c|}{9.01} & \multicolumn{2}{|c|}{3.3} \\
\hline & Ira & \multicolumn{2}{|c|}{10.5} & \multicolumn{2}{|c|}{3.6} & \multicolumn{2}{|c|}{10.7} & \multicolumn{2}{|c|}{3.8} \\
\hline & Hostilidad & \multicolumn{2}{|c|}{12.80} & \multicolumn{2}{|c|}{4.1} & \multicolumn{2}{|c|}{12.85} & \multicolumn{2}{|c|}{4.1} \\
\hline & Total & \multicolumn{2}{|c|}{50.54} & \multicolumn{2}{|c|}{11.7} & \multicolumn{2}{|c|}{47.14} & \multicolumn{2}{|c|}{12.42} \\
\hline & & \multicolumn{8}{|c|}{ Grupos de edad } \\
\hline & & \multicolumn{2}{|c|}{8 a 10 años } & \multicolumn{2}{|c|}{11 a 13 años } & \multicolumn{2}{|c|}{14 a 16 años } & \multicolumn{2}{|c|}{ Total } \\
\hline & & Media & $D E$ & Media & $D E$ & Media & $D E$ & Media & $D E$ \\
\hline \multirow{5}{*}{ 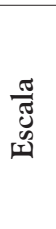 } & Física & 15.17 & 6.2 & 16.1 & 5.4 & 16.69 & 5.6 & 16.26 & 5.6 \\
\hline & Verbal & 8.69 & 3.1 & 8.94 & 2.9 & 9.38 & 3.4 & 9.12 & 3.2 \\
\hline & Ira & 10.14 & 3.6 & 10.43 & 3.6 & 10.92 & 3.7 & 10.62 & 3.6 \\
\hline & Hostilidad & 13.14 & 4 & 13.03 & 4.1 & 12.54 & 4.2 & 12.81 & 4.1 \\
\hline & Total & 47.16 & 12.8 & 48.52 & 12.1 & 49.54 & 12.1 & 48.83 & 12.1 \\
\hline
\end{tabular}

Fuente: elaboración propia. 


\section{TABLA 4}

Coeficiente a Cronbach e intervalos de confianza al 95\% para las escalas de la adaptación colombiana del AQ para los grupos de edad

\begin{tabular}{|c|c|c|c|c|c|}
\hline & & \multicolumn{4}{|c|}{ Grupos de edad } \\
\hline & & 8 a 10 años & 11 a 13 años & 14 a 16 años & Total \\
\hline \multirow{5}{*}{ 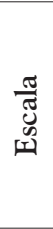 } & Física & $0.80(0.72-0.86)$ & $0.73(0.66-0.78)$ & $0.77(0.72-0.81)$ & $0.75(0.72-0.78)$ \\
\hline & Verbal & $0.48(0.27-0.64)$ & $0.51(0.39-0.60)$ & $0.66(0.59-0.73)$ & $0.58(0.52-0.63)$ \\
\hline & Ira & $0.36(0.11-0.52)$ & $0.58(0.46-0.64)$ & $0.63(0.55-0.70)$ & $0.58(0.52-0.63)$ \\
\hline & Hostilidad & $0.61(0.47-0.73)$ & $0.60(0.51-0.68)$ & $0.65(0.57-0.72)$ & $0.62(0.57-0.65)$ \\
\hline & Total & $0.82(0.75-0.88)$ & $0.81(0.77-0.85)$ & $0.83(0.79-0.86)$ & $0.82(0.80-0.84)$ \\
\hline
\end{tabular}

Fuente: elaboración propia.

dentro cada uno de los intervalo de confianza de estas escalas.

\section{Discusión}

Los resultados presentados en este estudio señalan la adecuación del AQ en su adaptación colombiana para preadolescentes y adolescentes, a la estructura factorial descrita para otros idiomas y adaptaciones tanto para preadolescentes y adolescentes (Ang, 2007; Santisteban \& Alvarado, 2009), como para adultos (Bryant \& Smith, 2001; Buss \& Perry, 1992; Vigil-Colet et al., 2005). Además, el buen ajuste del modelo logrado después de las numerosas modificaciones llevadas a cabo sobre los ítems de la versión española, va en línea con otros trabajos que han demostrado la importancia de no perder de vista las peculiaridades lingüísticas y culturales del entorno en que se aplican los cuestionarios (Chahín, Cosi, Lorenzo-Seva \& Vigil-Colet, 2010; Hambleton, 2005). De este modo, esta versión puede convertirse en un instrumento valioso, útil y práctico a la hora de evaluar los niveles de agresividad con fines diagnósticos o preventivos.

Con referencia a la fiabilidad, la presente adaptación ha mostrado que la escala total y la agresividad física poseen coeficientes adecuados para toda la muestra y para cada uno de los grupos de edad. Sin embargo, hay que considerar que en el grupo de menor edad ( 8 - 10 años), solo la escala de total y la de agresividad física son aptas para ser aplicadas, ya que las otras exhiben unos valores relativamente bajos. Por otra parte, en el grupo de mayor edad (14 -16 años) la confiabilidad de las es- calas de agresividad verbal, ira y hostilidad tiende a mejorar, presentando coeficientes marginalmente aceptables. Cabe señalar que estos valores en los distintos grupos de edad no son exclusivos de la adaptación colombiana, pues resultados similares con muestras de adolescentes y preadolescentes han sido reportados, tanto en versiones largas adaptadas a esta población (Santisteban \& Alvarado, 2009), como en versiones reducidas (Ang, 2007). Es preciso resaltar que la menor confiabilidad de las escalas de agresividad verbal, ira e y hostilidad ha sido una constante desde que Buss y Perry (1992) desarrollaron la primera versión del $\mathrm{AQ}$, en la que reportaron confiabilidades alrededor de $a=0.7$ para dichas escalas, mientras que para la escala total y la de agresividad física se situó en $\boldsymbol{\alpha}=0.8$, obteniéndose los mismos valores para la versión reducida española a partir de la cual se ha desarrollado la adaptación colombiana (Morales-Vives et al., 2005). Algunos estudios incluso han señalado confiabilidades en adultos notablemente inferiores a las reportadas por nosotros en adolescentes y preadolescentes. Así Meesters et al. (1996) y García-León et al. (2002) reportaron una $a=0.5$ para la escala de agresividad verbal en la adaptación holandesa y española, respectivamente. Todo ello indica que desde el punto de vista de la confiabilidad, la adaptación colombiana puede considerarse equivalente a las existentes en otros idiomas cuando se aplica a población adolescente y, ligeramente inferior en algunas escalas en población preadolescente.

No obstante lo anterior, diversos estudios han señalado que en general los datos obtenidos mediante autoinformes en niños a partir de 8 años, a 
pesar de presentar fiabilidades relativamente bajas, suelen ser mejores indicadores de su comportamiento que aquellos respondidos por sus padres y profesores (Achenbach, McConaughy \& Howell, 1987; Cosi, Canals, Hernández-Martínez \& Vigil-Colet, 2010; Muris, Meesters \& Schouten, 2002). Es por esto que a pesar de la baja fiabilidad de las escalas de agresividad verbal, ira y hostilidad, no se descartaría la utilización de todas las escalas del cuestionario entre los 8 y 14 años. En último término, para estas edades podría complementarse la información proporcionada por el AQ con otros cuestionarios en español, que son los aplicados a sus profesores o padres, como el Test de Agresividad Proactiva/ Reactiva (Cosi, Vigil-Colet \& Canals, 2009).

$\mathrm{Al}$ analizar las diferencias de agresividad entre sexos, se ha verificado también que esta adaptación colombiana del AQ para preadolescentes y adolescentes es sensible a las diferencias en agresividad física. Esto mismo ha sido reportado en numerosas investigaciones, en el sentido que los varones presentan niveles de agresividad física más altos que las mujeres, en un amplio rango de edades que va desde la adolescencia hasta la tercera edad (Archer, 2004; Morales-Vives \& Vigil-Colet, 2010). Este resultado aporta una primera evidencia de validez de constructo para la presente versión. Cabe resaltar que el tamaño de efecto encontrado para esta diferencia $(d=0.64)$ es similar al reportado en otras investigaciones en población adolescente como las de Santisteban y Alvarado (2009) o Vigil-Colet et al. (2008) que presentaron tamaños del efecto de $d=0.69 \mathrm{y} d=0.47$, respectivamente. De este modo, las diferencias en agresividad física en esta muestra colombiana pueden considerarse equivalentes a las encontradas en adolescentes españoles.

A partir de los resultados aquí presentados, se abre una primera línea de acción para futuras investigaciones que debe aportar evidencia sobre el grado de validez convergente del AQ en estas edades con otros cuestionarios y dimensiones de personalidad que han sido frecuentemente relacionadas con la agresividad, así como sobre su validez predictiva en relación con los comportamientos violentos. Del mismo modo, una segunda línea debe permitir en el futuro llevar a cabo comparaciones interculturales sobre los niveles de agresividad, donde se pueda establecer si las distintas adaptaciones están midiendo el constructo de la misma manera. Este es un aspecto clave a la hora de llevar a cabo comparaciones transculturales y se relaciona con el establecimiento de una serie de igualdades jerárquicas entre las distintas adaptaciones (Byrne, 2008; Byrne \& Watkins, 2003). Estas igualdades se refieren al grado en que las distintas adaptaciones presentan los mismos factores e ítems (invarianza configuracional), al grado en que la relación entre las variables latentes y los ítems es equivalente (igualdad de saturaciones factoriales o invarianza factorial débil) y, finalmente, la invarianza factorial fuerte en el caso de igualdad de los interceptos en la relación entre las variables latentes y los ítems (Meredith, 1993). Tan solo el establecimiento de estas tres igualdades garantiza que las posibles diferencias entre, por ejemplo, la versión española y la colombiana, sean debidas a auténticas diferencias en agresividad y no a un funcionamiento diferencial de los ítems. Con referencia a lo expuesto anteriormente, el presente trabajo ha establecido que la estructura factorial y la composición de los factores del AQ es la misma en las versiones colombiana y española, aportando evidencia sobre la invarianza configuracional. Con todo lo señalado, si se desea llevar a cabo en el futuro comparaciones entre las puntuaciones del AQ entre ambos países, sería necesario utilizar muestras de edad y nivel sociocultural equivalentes.

\section{Referencias}

Achenbach, T. M., McConaughy, S. H. \& Howell, C. T. (1987). Child/adolescent behavioral and emotional problems: Implications of cross-informant correlations for situational specificity. Psychological Bulletin, 101, 213-232.

Andreu, J. M., Peña, M. E. \& Graña, J. L. (2002). Adaptación psicométrica de la versión española del Cuestionario de Agresión. Psicothema, 14, 476-482.

Ang, R. (2007). Factor structure of the 12-item Aggression Questionnaire: Further evidence from Asian adolescent samples. Journal of Adolescence, 30, 671-685. 
Archer, J. (2004). Sex differences in real-word settings: A meta-analytic review. Review of General Psycho$\log y, 8,291-332$.

Bentler, P. M. (1990). Comparative fit indices in structural models. Psychological Bulletin, 107, 238-246.

Bernstein, I. H. \& Gesn, P. R. (1997). On the dimensionality of the Buss/Perry Aggression Questionnaire. Behavioral Research and Therapy, 35, 563-568.

Bryant, F. B. \& Smith, B. D. (2001). Refining the architecture of aggression: A measurement model for the Buss-Perry Aggression Questionnaire. Journal of Research on Personality, 35, 138-167.

Buss, A. H. \& Durkee, A. (1957). An inventory for assessing different kinds of hostility. Journal of Consulting Psychology, 21, 343-349.

Buss, A. H. \& Perry, M. P. (1992). The Aggression Questionnaire. Journal of Personality and Social Psychology, 63, 452-459.

Byrne, B. M. (2008). Testing for multigroup equivalence of a measuring instrument: A walk through the process. Psichothema, 20, 872-882.

Byrne, B. M. \& Watkins, D. (2003). The issue of measurement invariance revisited. Journal of CrossCultural Psychology, 34, 155-175.

Castrillón, D. A., Ortiz, P. A. \& Vieco, F. (2004). Cualidades paramétricas del Cuestionario de Agresión (AQ) de Buss y Perry en estudiantes universitarios de la ciudad de Medellín (Colombia). Revista Facultad Nacional de Salud Pública, 22, 49-61.

Chahín, N., Cosi, S., Lorenzo-Seva, U. \& Vigil-Colet, A. (2010). Stability of the factorial structure of Barrat's Impulsivity Scales for children across cultures: A comparison between Spain and Colombia. Psicothema, 22, 983-989.

Condon, L., Morales-Vives, F., Ferrando, P. J. \& VigilColet, A. (2006). Sex differences in the full and reduced versions of the Aggression Questionnaire: A question of differential item functioning? European Journal of Psychological Assessment, 22, 92-97.

Cosi, A., Vigil-Colet, A. \& Canals, J. (2009). Desarrollo del Cuestionario de Agresividad proactiva/reactiva para profesores: estructura factorial y propiedades psicométricas. Psicothema, 21, 159-164.

Cosi, A., Canals, J., Hernández-Martínez, C. \& VigilColet, A. (2010). Parent-child agreement in SCA-
RED and its relationship to anxiety symptoms. Journal of Anxiety Disorders, 24, 129-133.

Fan, X. \& Sivo, S. A. (2007). Sensitivity of fit indices to model misspecification and model types. Multivariate Behavioral Research, 42, 509-529.

Fossati, A., Maffei, C., Acquarini, E. \& Di Ceglie, A. (2003). Multigroup confirmatory component and factor analyses of the Italian version of the Aggression Questionnaire. European Journal of Psychological Assessment, 19, 54-65.

García-León, A., Reyes, G. A., Vila, J., Pérez, N., Robles, H. \& Ramos, M. M. (2002). The Aggression Questionnaire: A validation study in student samples. The Spanish Journal of Psychology, 5, 45-53.

Hambleton, R. K. (1994). Guidelines for adapting educational and psychological tests: A progress report. European Journal of Psychological Assessment, 10, 229-244.

Hambleton, R. K. (2005). Issues, designs, and technical guidelines for adapting tests into multiple languages and cultures. In R. K. Hambleton, P. F. Merenda \& C. Spielberger (Eds.), Adapting educational and psychological tests for cross-cultural assessment (pp. 3-38). London: L.E.A.

Harris, J. A. (1995). Confirmatory factor analysis of the Aggression Questionnaire. Behavioral Research and Therapy, 8, 991-993.

Hu, L. \& Bentler, P. M. (1999). Cut off criteria for fit indexes in covariance structure analysis: Conventional criteria versus new alternatives. Structural Equation Modeling, 6, 1-55.

Lovas, L. \& Trenkova, S. (1996). Aggression and perception of an incident. Studia Psychologia, 38, 265-270.

Meesters, C., Muris, P., Bosma, H., Schouten, E. \& Beuving, S. (1996). Psychometric evaluation of the Dutch version of the Aggression Questionnaire. Behavioral Research and Therapy, 34, 839-843.

Meredith, W. (1993). Measurement invariance, factor analysis and factorial invariance. Psychometrika, $58,525-543$.

Morales-Vives, F., Codorniu-Raga, M. J. \& Vigil-Colet, A. (2005). Psychometric properties of the reduced versions of Buss and Perry's Aggression Questionnaire. Psicothema, 17, 96-100. 
Morales-Vives, F. \& Vigil-Colet, A. (2010). Are there sex differences in physical aggression in the elderly? Personality and Individual Differences, 49, 659-662.

Muris, P., Meesters, C. \& Schouten, E. (2002). A brief questionnaire of DSM-IV-Defined anxiety and depression symptoms among children. Clinical Psychology and Psychotherapy, 9, 430-442.

Muthen, L. K. \& Muthen, B. O. (2008). Mplus (Version 5.1). Los Angeles, CA: Muthen \& Muthen.

Nakano, K. (2001). Psychometric evaluation on the Japanese adaptation of the Aggression Questionnaire. Behavioral Research and Therapy, 39, 853-858.

Pfister, R. \& Masse, P. (2001, noviembre). Le Questionnaire d'Agressivité: validation et équivalence transculturelle. IXème Congrès International des Chercheurs en Activités Physiques et Sportives, Valence, France.

Ramírez, J., Andreu, J. M. \& Fujihara, T. (2001). Cultural and sex differences in aggression: A comparison between Japanese and Spanish students using two different inventories. Aggressive Behavior, 27, 313-322.

Santisteban, C. \& Alvarado, J. M. (2009). The Aggression Questionnaire for Spanish preadolescents and adolescents: PA-AR. The Spanish Journal of Psychology, 12, 320-326.

Santisteban, C., Alvarado, J. M. \& Recio, P. (2007). Evaluation of a Spanish version of the Buss ansd Perry Agression Questionnaire: Some personal and situational factors related to the aggression scores of young subjects. Personality and Individual Differences, 42, 1453-1462.

Sierra, J. C. \& Gutiérrez, J. R. (2007). Validación de la versión española del Cuestionario de Agresión de Buss-Perry en estudiantes universitarios salvadoreños. Psicología y Salud, 107, 103-113.

van de Vijver, F. J. R. \& Hambleton, R. K. (1996). Translating tests: Some practical guidelines. European Psychologist, 1, 89-99.

Vigil-Colet, A., Lorenzo-Seva, U., Codorniu-Raga, M. J. \& Morales, F. (2005). Factor structure of the Aggression Questionnaire among different samples and languages. Aggressive Behavior, 31, 601-608.

Von Collani, G. \& Werner, R. (2005). Self-related and motivational constructs as determinants of aggression. An analysis and validation of a German version of the Buss-Perry Aggression Questionnaire. Personality and Individual Differences, 38, 1631-1643. 